\title{
The impact of short and long sleep duration on instrumental activities of daily living among stroke survivors
}

\author{
Azizi A Seixas ${ }^{1,2, *}$ \\ Debbie P Chungl,* \\ Shannique L Richards' \\ Shreya Madhavaram' \\ Preeti Raghavan ${ }^{1,3}$ \\ Juan Gago 1,4 \\ George Casimir ${ }^{1,5}$ \\ Girardin Jean-Louis ${ }^{1,2}$ \\ 'Department of Population Health, \\ Center for Healthful Behavior Change, \\ NYU Langone Health, NY, New York, \\ USA; ${ }^{2}$ Department of Psychiatry, \\ Center for Healthful Behavior \\ Change, NYU Langone Health, NY, \\ New York, USA; ${ }^{3}$ Department of \\ Rehabilitation Medicine, NYU Langone \\ Health, NY, New York, USA; ${ }^{4}$ Cervical \\ Cancer Prevention Program, National \\ Cancer Institute of Argentina, Buenos \\ Aires, Argentina; ${ }^{5}$ Department of \\ Psychiatry, SUNY Downstate Medical \\ Center, Brooklyn, NY, USA \\ *These authors contributed equally \\ to this work
}

This article was published in the following Dove Press journal: Neuropsychiatric Disease and Treatment

\begin{abstract}
Objective: Stroke survivors generally have problems completing instrumental activities of daily living (IADL; eg, preparing meals, chores, taking a bath, and managing finances). However, it is unclear how stroke survivors might stave off IADL issues. Studies indicating that sleep has restorative neurological effects provide potential mechanisms to address issues with IADL. The aim of this study was to ascertain the association between sleep duration (short or long sleep duration) and IADL among stroke survivors and those without a stroke history.
\end{abstract}

Methods: Data of 486,619 participants were analyzed from the 2000 to 2015 National Health Interview Survey (NHIS), a nationally representative sample. Measures of self-reported stroke, sociodemographic variables, sleep duration, and IADL problems were collected. Binary logistic regression was utilized to analyze the relationship of short ( $\leq 6$ hours) and long ( $\geq 9$ hours) sleep duration with limitations to IADL.

Results: Of the sample, 3\% reported a physician-diagnosed stroke event. The mean age was 45.73 years; $52.7 \%$ were female; $77.4 \%$ were White; $14.2 \%$ were Black; $41.3 \%$ were married, $62.7 \%$ were employed; $31.1 \%$ reported that annual family income was less than $\$ 35,000 ; 87 \%$ reported good-to-excellent health; and $29.7 \%$ reported short sleep ( $\leq 6$ hours). Approximately $30 \%$ of stroke survivors reported IADL problems, and $34.4 \%$ who reported IADL problems were short sleepers. Among stroke survivors, long sleepers were $97 \%$ more likely than average sleepers to report IADL problems $(\mathrm{OR}=1.97,95 \% \mathrm{CI}=1.71-2.26, P<0.001)$ adjusting for age, sex, race, marital status, poverty, and health.

Conclusion: Findings from our study indicate that, among stroke survivors, long sleepers were more likely to report IADL problems compared to average sleepers (7-8 hours). Future studies should investigate other potential mediators such as severity of stroke, medication, comorbidities, level of impairment, and whether improving sleep among stroke survivors may improve IADL.

Keywords: stroke, sleep, instrumental activities of daily living, quality of life, functional impairment

\section{Introduction}

According to the Centers for Disease Control and Prevention (CDC), stroke affects approximately 800,000 individuals each year and is the leading cause of serious long-term disability in USA. Besides physical impairment, stroke survivors often suffer from cognitive impairment (mild or severe), fatigue and sleep disturbances, mental health issues, and diminished quality of life, all of which can contribute to difficulty with instrumental activities of daily living (IADL), such as preparing meals, completing chores, taking a shower, or managing finances. ${ }^{1,2}$ Being able to perform
Correspondence: Azizi A Seixas Department of Population Health, Center for Healthful Behavior Change, NYU Langone Health, I80 Madison Avenue, New York, NY 10016, USA Email azizi.seixas@nyulangone.org
Neuropsychiatric Disease and Treatment 2019:15 177-182

177

Dovepress $f$ in 0

http:

(c) (1) (2) 2019 Seixas et al. This work is published and licensed by Dove Medical Press Limited. The full terms of this license are available at https://www.dovepress.com/terms.php
and incorporate the Creative Commons Attribution - Non Commercial (unported, v3.0) License (http://creativecommons.org/licenses/by-nc/3.0/). By accessing the work you (c)
hereby accept the Terms. Non-commercial uses of the work are permitted without any further permission from Dove Medical Press Limited, provided the work is properly attributed. For permission for commercial use of this work, please see paragraphs 4.2 and 5 of our Terms (https://www.dovepress.com/terms.php). 
IADL is necessary for successful independent living, and an inability to perform IADL among stroke survivors is associated with increased mortality, stroke recurrence, slower/ poorer recovery, and greater levels of functional impairment, after adjusting for vascular risk factors. ${ }^{3}$ A better understanding of the causes for impairment in IADL is needed to offer potential remediation strategies during rehabilitation and improve stroke recovery.

Fatigue and sleep disturbances (notably short or long sleep durations, poor sleep quality, and sleep disorders such as insomnia and obstructive sleep apnea) are common after a stroke. ${ }^{4-6}$ These sleep-related health conditions are linked to poor psychosocial outcomes such as decreased likelihood of returning to paid employment 12 months after a stroke after adjusting for age, sex, and mental health comorbidity. ${ }^{7}$ This finding calls for a deeper understanding of the impact of sleep problems on functional outcomes among stroke survivors, specifically on global functional outcomes such as IADL.

Sleep duration has been shown to be strongly associated with poor functional outcomes and neurocognitive dysfunction among stroke survivors. ${ }^{8,9}$ Short sleep duration $(<7$ hours per 24-hour period) has been linked to various functional difficulties such as driving, taking care of financial affairs, or performing work-related tasks. ${ }^{10,11}$ Long sleep duration ( $>8$ hours) is also linked to impaired functional outcomes. ${ }^{4}$ Both short and long sleep duration may be caused by insomnia and sleep apnea which can lead to increased daytime sleepiness in stroke patients. ${ }^{12-14}$

Therefore, the aim of the current study was to determine the association between sleep duration and daily functioning, particularly with respect to performing IADL, among stroke survivors and individuals without a stroke history.

\section{Methods}

We analyzed data from the 2000 to 2015 National Health Interview Survey (NHIS), a nationally representative multipurpose health survey of civilian, noninstitutionalized US residents, conducted by the CDC's National Center for Health Statistics (NCHS). The data collected in the NHIS are obtained through a complex, multistage sample design that involves stratification, clustering, and oversampling of specific population subgroups, such as minority populations. Details on sample design are reported elsewhere. ${ }^{15,16}$ Trained staff from the US Census Bureau collected data according to procedures specified by the NCHS in face-to-face interviews using computer-assisted personal interviewing methods as well as telephone surveys. Respondents during the face-toface interviews provide sociodemographic data (age, sex, race/ethnicity, average family income, and education), health risks (smoking status and alcohol intake), and physiciandiagnosed chronic conditions or diseases (hypertension, diabetes, coronary heart disease, cancer, kidney disease, stroke, and myocardial infarction). This study was carried out in accordance with the recommendations of CDC with written informed consent from all subjects and in accordance with the Declaration of Helsinki. The protocol was approved by the CDC.

\section{Target variables and data analysis}

We used sociodemographic, behavioral/lifestyle, psychosocial, and self-reported medical data from the NHIS spanning 15 consecutive years (2000-2015). All information collected was self reported.

\section{Problems with IADL}

The ability to perform IADL was assessed by whether an individual "currently needed the help of other persons in handling routine needs, such as everyday household chores, doing necessary business, shopping, or getting around for other purposes, because of a physical, mental, or emotional problem." Participants were asked to report if they needed help or not. Individuals who reported "no" received a score of 1 and those who reported "yes" received a score of " 2 ".

\section{History of stroke}

Individuals were asked whether they ever had a physiciandiagnosed stroke. "No" responses were coded as 1 , and "yes" responses were coded as 2 .

\section{Self-reported sleep duration}

Participants were asked, "How many hours of sleep do you get on average in a 24-hour period?" We classified sleep duration into three categories: short ( $\leq 6$ hours), average (7-8 hours), and long ( $\geq 9$ hours) sleep duration.

The data were analyzed using IBM SPSS Statistics for Windows (version 20.0; IBM Corporation, Armonk, NY, USA). Binary logistic regression was utilized to analyze the relationship between sleep duration and limitations in IADL among stroke survivors and among individuals who did not report a history of stroke (we built two regression models). In developing the regression model, we conducted several analytical steps which included the following: 1) assessing whether independent and dependent variables (sleep and IADL, respectively) as well as covariates were normally distributed and if they had high levels of skewness and kurtosis $( \pm 2)$; 2) determining the correlation between independent, dependent, and covariate variables; 3 ) if variables were 
associated with either independent or dependent variables, they were deemed as confounders and we adjusted for their potentially confounding effects in the regression model; 4) in addition to empirically determining covariate candidates, we also searched existing literature that indicated strong associations between our independent and dependent variables. Based on our empirical and content search, we identified age, sex, marital status, self-reported health, and poverty as covariates for our regression model. Although the extant literature suggests that depression is a potential confounder, our dataset did not assess for depression over the time frame of 2000-2015, and so we were unable to test for an association.

\section{Covariates}

The factors such as age, sex, marital status, self-reported health status (self-reported health status is defined as an individual's general self-reported health, and individuals report it along a Likert scale from "excellent" to "poor"), and poverty status (the ratio of family income to the US Census Bureau's poverty thresholds for the year) were utilized as covariates.

\section{Results}

\section{Descriptive statistics}

The original sample consisted of $1,108,043$ participants; however, only 486,619 participants (43.9\%) provided a history of stroke; therefore, only these participants were included in the final sample for the regression analyses. A statistical description of the sample is provided in Table 1. The mean age of the sample was 45.7 years ( $\mathrm{SD}=14.15$ ); $51.1 \%$ identified themselves as female, $77.4 \%$ as White, $14.2 \%$ as Black, $41.3 \%$ as married, $62.7 \%$ reported that they were currently working, $31.1 \%$ had families earning less than $\$ 35,000$ annually, and $90.3 \%$ reported that their general health status was within the "good-to-excellent" range. Approximately 3\% of the sample reported a physician-diagnosed stroke, $29.8 \%$ reported short sleep duration ( $<7$ hours), $61.65 \%$ reported average sleep duration (7-8 hours), 8.6\% reported long sleep duration ( $>8$ hours), and $3.9 \%$ reported that they needed help or had issues with IADL. Regarding the need for help with IADL based on stroke status, $29.2 \%$ of stroke survivors reported that that needed help with IADL, while only $4.2 \%$ of individuals without a stroke history needed help with IADL. With regard to habitual sleep status and need for help with IADL, 34.4\% of respondents who reported problems with IADL were short sleepers, while $24.5 \%$ who reported problems with IADL were long sleepers.
Table I Sociodemographic characteristics of survey participants

\begin{tabular}{l|l}
\hline Variables & $\begin{array}{l}\text { Percentage of } \\
\text { participants }\end{array}$ \\
\hline Female & 52.70 \\
Ethnicity & 77.40 \\
$\quad$ White & 14.20 \\
$\quad$ Black & 41.30 \\
Married & 62.70 \\
Currently working & 31.10 \\
Families earning $<\$ 35,000$ annually & 87.00 \\
Self-reported general health status as "excellent & \\
to good" & \\
Self reported & 3.00 \\
Physician-diagnosed stroke & \\
Sleep duration & 29.84 \\
$\quad$ Short sleep duration ( $<7$ hours) & 61.57 \\
$\quad$ Average sleep (7-8 hours) & 8.58 \\
$\quad$ Long sleep duration ( $>8$ hours) & 3.87 \\
Difficulties with IADL & 29.16 \\
Individuals with stroke history who had & \\
difficulties with IADL & 4.22 \\
Individuals with no stroke history who had & \\
difficulties with IADL & Individuals with difficulties with IADL who \\
were short sleepers & 34.40 \\
Individuals with difficulties with IADL who & 24.50 \\
were long sleepers & \\
\hline Abbreviation IADL instion &
\end{tabular}

Abbreviation: IADL, instrumental activities of daily living.

Specifically among stroke survivors, $57.7 \%$ were female, $8.6 \%$ reported being married and living with a spouse, $71.3 \%$ reported not working in the past 12 months, $19.9 \%$ reported poor health status, $29.4 \%$ reported a history of physiciandiagnosed diabetes, $26 \%$ reported a history of heart disease, and $73.8 \%$ reported a history of hypertension. The highest stroke prevalence was in the 45-64 (32.50\%) and 75-85 (35.20\%) age groups.

\section{Inferential statistics}

Results from binomial logistic regression analyses testing the independent association between sleep duration and IADL among stroke survivors and non-stroke survivors indicated that, among stroke survivors, long sleepers were $97 \%$ more likely than average sleepers to report issues with IADL ( $\mathrm{OR}=1.97,95 \% \mathrm{CI}=1.71-2.26, P<0.001)$, after adjusting for the confounding effects of age, sex, race, marital status, self-reported health status, and poverty status (Table 2). Among individuals without a stroke history, both short and long sleep durations were predictive of IADL difficulty. Specifically, short sleepers were approximately $25 \%$ more likely than average sleepers to report issues with IADL ( $\mathrm{OR}=1.25$, 95\% CI $=1.18-1.30, P<0.001$ ), while long sleepers were two and a half times more likely to report issues with IADL 
Table 2 Independent association of sleep duration on IADL among stroke survivors

\begin{tabular}{|c|c|c|c|c|}
\hline \multicolumn{5}{|l|}{ OR estimates } \\
\hline \multirow[t]{2}{*}{ Effect } & \multirow[t]{2}{*}{$\begin{array}{l}\text { Point } \\
\text { estimate }\end{array}$} & \multicolumn{2}{|c|}{$\begin{array}{l}95 \% \text { confidence } \\
\text { limits }\end{array}$} & \multirow[t]{2}{*}{ P-value } \\
\hline & & Lower & Upper & \\
\hline \multicolumn{5}{|l|}{ Hours of sleep } \\
\hline 7-8 sleep hours (ref) & - & - & - & - \\
\hline Short sleeper ( $<6$ hours) & 0.90 & 0.79 & 1.03 & 0.12 \\
\hline$>8$ sleep hours* & 1.97 & 1.71 & 2.27 & $<0.00$ I \\
\hline Age* & 1.03 & 1.02 & 1.04 & $<0.00$ I \\
\hline \multicolumn{5}{|l|}{ Sex } \\
\hline Male (ref) & - & - & - & - \\
\hline Female* & 1.57 & 1.40 & 1.77 & $<0.000$ I \\
\hline \multicolumn{5}{|l|}{ Marital status } \\
\hline Married (ref) & - & - & - & - \\
\hline Never married* & 2.42 & 1.95 & 3.01 & $<0.001$ \\
\hline Separated* & 1.91 & $\mathrm{I} .42$ & 2.56 & 0.56 \\
\hline Unknown marital status & 1.39 & 0.45 & 4.29 & $<0.001$ \\
\hline Widowed* & 2.19 & 1.87 & 2.58 & $<0.001$ \\
\hline Divorced* & 1.62 & 1.39 & 1.88 & $<0.00$ I \\
\hline \multicolumn{5}{|l|}{ Self-reported } \\
\hline \multicolumn{5}{|l|}{ health status } \\
\hline \multicolumn{5}{|l|}{ Excellent (ref) } \\
\hline Very good* & 1.17 & 0.80 & 1.71 & 0.43 \\
\hline Good* & 2.65 & 1.81 & 3.87 & $<0.001$ \\
\hline Fair* & 6.39 & 4.39 & 9.31 & $<0.001$ \\
\hline Poor* & 16.35 & 11.15 & 23.97 & $<0.001$ \\
\hline \multicolumn{5}{|l|}{ Poverty status } \\
\hline \multicolumn{5}{|l|}{ At or above poverty (ref) } \\
\hline Below poverty & 1.44 & 1.24 & 1.66 & $<0.00$ I \\
\hline Unknown & 0.99 & 0.86 & 1.15 & 0.92 \\
\hline
\end{tabular}

Note: *Significant finding at $P<0.05$.

Abbreviations: IADL, instrumental activities of daily living; ref, reference.

compared to average sleepers $(\mathrm{OR}=2.66,95 \% \mathrm{CI}=2.50-2.82$, $P<0.001$ ) adjusting for the confounding effects of age, sex, race, marital status, self-reported health status, and poverty status (Table 3 ). In summary, long sleep duration among both stroke survivors and individuals without a stroke history was independently associated with IADL difficulty and had a stronger association compared to short sleep duration.

We also found that female stroke survivors were 57\% more likely to suffer from issues with IADL compared to their male counterparts. This finding is consistent with previous work that suggests that middle-aged female stroke survivors suffer from cognitive appraisal deficits, depression, low quality of life, and poor activities of daily living. ${ }^{17,18}$

\section{Discussion}

The current study examined the impact of sleep duration on difficulty with IADL among stroke survivors and individuals without a stroke history, adjusting for confounding effects of age, sex, marital status, self-reported health status, and
Table 3 Independent association of sleep duration on IADL among individuals without a stroke history

\begin{tabular}{|c|c|c|c|c|}
\hline \multicolumn{5}{|l|}{ OR estimates } \\
\hline \multirow[t]{2}{*}{ Effect } & \multirow[t]{2}{*}{$\begin{array}{l}\text { Point } \\
\text { estimate }\end{array}$} & \multicolumn{2}{|c|}{$\begin{array}{l}\text { 95\% confidence } \\
\text { limits }\end{array}$} & \multirow[t]{2}{*}{$P$-value } \\
\hline & & Lower & Upper & \\
\hline \multicolumn{5}{|l|}{ Hours of sleep } \\
\hline 7-8 sleep hours (ref) & - & - & - & - \\
\hline Short sleeper ( $<6$ hours) & 1.25 & 1.18 & 1.31 & $<0.001$ \\
\hline$>8$ sleep hours* & 2.66 & 2.50 & 2.82 & $<0.001$ \\
\hline Age* & 1.05 & 1.04 & 1.05 & $<0.001$ \\
\hline \multicolumn{5}{|l|}{ Sex } \\
\hline Male (ref) & - & - & - & - \\
\hline Female* & 1.48 & 1.14 & 1.56 & $<0.001$ \\
\hline \multicolumn{5}{|l|}{ Marital status } \\
\hline Married (ref) & - & - & - & - \\
\hline Never married* & 2.83 & 2.61 & 3.06 & $<0.001$ \\
\hline Separated* & 1.91 & 1.69 & 2.15 & $<0.001$ \\
\hline Unknown marital status & 1.10 & 0.72 & 1.68 & 0.659 \\
\hline Widowed* & 2.46 & 2.30 & 2.64 & $<0.001$ \\
\hline Divorced* & 1.90 & 1.77 & 2.03 & $<0.001$ \\
\hline \multicolumn{5}{|l|}{ Self-reported } \\
\hline \multicolumn{5}{|l|}{ health status } \\
\hline \multicolumn{5}{|l|}{ Excellent (ref) } \\
\hline Very good* & 1.78 & 1.57 & 2.00 & $<0.001$ \\
\hline Good* & 4.90 & 4.38 & 5.50 & $<0.001$ \\
\hline Fair* & 16.33 & 14.55 & 18.33 & $<0.001$ \\
\hline Poor* & 59.65 & 52.96 & 67.19 & $<0.001$ \\
\hline
\end{tabular}

Note: *Significant finding at $P<0.05$.

Abbreviations: IADL, instrumental activities of daily living; ref, reference.

poverty status. Although previous studies indicate that short sleep is associated with negative poststroke health outcomes, findings from our current study indicate that long sleep duration rather than short sleep duration is more strongly associated with IADL difficulty among stroke survivors. Our findings also indicate that, among individuals without a history of stroke, both short and long sleep durations were independently associated with IADL difficulty. The neurobiological and biobehavioral explanations for our findings are discussed in the following paragraphs.

Our findings are consistent with previous studies which found in unadjusted models that short and long sleep durations were associated with IADL difficulty in a sample of $60+$ year olds in Spain. ${ }^{17}$ Specifically, compared to average sleepers, short sleepers were $56 \%$ more likely and long sleepers were $108 \%$ more likely to report difficulty with IADL. Interestingly, only the relationship between long sleep and IADL remained significant after adjusting for medical comorbidity and sleep quality. For our study, we found a similar pattern where long sleep duration, not short sleep duration, remained significant in predicting IADL difficulty among stroke survivors. Older individuals are 
more likely to have vascular disease and mild cognitive impairment, with or without a stroke, which can affect their ability to successfully perform IADL. ${ }^{18,19}$ Longer sleep duration in these individuals may therefore be a risk indicator of undiagnosed neurodegenerative disease or early dementia. In the case of stroke survivors, long sleep duration may suggest increased risk for a secondary stroke or a corollary comorbidity, due to more extensive cerebrovascular disease.

In addition to the neurobiological explanation of our findings, there may also be a biobehavioral explanation. Since short sleep duration was no longer significant in adjusted models whereas long sleep duration was, it may be that long sleep duration is truly independently associated with IADL difficulty. The conventional scientific view is that the effect of long sleep duration on health outcomes, especially IADL difficulty, is due to a synergistic effect - that is, its impact on health outcomes is coupled by another driving force, such as a medical comorbidity, depression, increased inflammation, pain, obesity, diabetes, and heart disease where they are all common among stroke survivors. ${ }^{20-22}$ Our results, after adjusting for comorbidity, however, suggest otherwise and argue that the effect of long sleep duration on IADL difficulty may be more direct than synergistic or additive effect.

Findings from our study have implications for how stroke survivors are medically treated and may provide insight about the prognosis for recovery and rehabilitation after a stroke. Moreover, our findings are consequential for stroke survivors, because sleep may play a critical role in recovery as previous work links sleep to neurorehabilitation and learning which may be compromised after a stroke. ${ }^{23}$ Therefore, the lack of optimal sleep (short or long sleep duration) may prevent or retard neurological reorganization after a stroke which in turn may compromise cognitive functioning and stroke survivors' ability to perform or relearn IADL successfully.

\section{Study limitations}

Results from our study should be interpreted cautiously in light of the following potential limitations. First, selfreported sleep duration, as opposed to objective measurement of sleep captured by polysomnography or actigraphy, may not be the most accurate measurement of habitual sleep duration. Second, the cross-sectional design of the NHIS dataset precludes making causal inferences between sleep duration and IADL. Third, self-reported sleep duration is limited and unreliable because studies do not distinguish between weekday and weekend sleep, which typically varies. Fourth, our regression models did not test the potential confounding role of depression on the relationship between sleep duration and IADL, because our dataset did not assess for depression. Fifth, although our dichotomization of the IADL measure is widely accepted especially in the NHIS dataset, it is not ideal because it misses potentially meaningful nuances if it were to be assessed dimensionally. Finally, we were unable to determine the type of stroke, number of strokes, duration of stroke, chronicity/severity of stroke, functional status as measured by modified Rankin Scale, medical comorbidities, and medication and physical function after the stroke which could influence (statistical confounders) an individual's ability to perform IADL. Future studies should investigate whether reduction in sleep duration from long sleep to average sleep (7-8 hours), perhaps through interventions targeted at improving sleep quality and efficiency, could improve IADL among stroke survivors. Second, future studies should investigate whether sleep duration affects differential IADL rates between men and women. Finally, future studies should use more robust measures and test a more comprehensive model with more covariates such as the level of impairment, nutrition, physical activity amount, and intensity performed.

\section{Conclusion}

Stroke survivors, who report habitual long sleep duration as opposed to short sleep duration, are more likely to report problems with IADL. Conversely, both short and long sleep durations were independently associated with IADL difficulty among individuals without a stroke history. There are neurobiological and biobehavioral explanations for these findings as well as consequences for treatment and rehabilitation of stroke survivors. Improving overall sleep quality may improve IADL functioning after a stroke. We argue that stroke survivors should be screened for underlying sleep problems (specifically long sleep duration) and stroke survivors should be encouraged to get necessary treatment for sleep disturbances as it may improve their quality of life and assist them in their recovery.

\section{Acknowledgments}

This study was supported by funding from the National Heart Lung, Blood and Sleep Institute (K01HL135452), National Institute of Minority Health Disparity (R01MD007716), and National Institute of Child Health and Human Development (R01HD071978). However, the funders had no role in study design, data collection and analysis, decision to publish, or preparation of the manuscript. An abstract of this paper was 
presented at the Associated Professional Sleep Societies (APSS) 31st Annual Meeting, June 3-7, 2017, Boston, MA, USA, as a poster presentation with interim findings. The poster's abstract has been published. ${ }^{24}$

\section{Author contributions}

AAS and DPC developed the idea of the paper, led the paper, and played key roles in the development of all components of the manuscript. SLR, SM, and PR developed the "Introduction" section, JG and AAS performed the analyses. AAS, DPC, GC, FZ, PR, and GJL developed and edited the "Discussion" section. All authors contributed toward data analysis, drafting and critically revising the paper, gave final approval of the version to be published, and agree to be accountable for all aspects of the work.

\section{Disclosure}

The authors report no conflicts of interest in this work.

\section{References}

1. Carlsson G. Mild Stroke Consequences in Everyday Life, Coping and Life Satisfaction. Göteborg: Institute of Neuroscience and Physiology;2007.

2. Carlsson GE, Möller A, Blomstrand C. Consequences of mild stroke in persons $<75$ years - a 1-year follow-up. Cerebrovasc Dis. 2003;16(4): 383-388.

3. Swartz RH, Bayley M, Lanctôt KL, et al. Post-stroke depression, obstructive sleep apnea, and cognitive impairment: Rationale for, and barriers to, routine screening. Int J Stroke. 2016;11(5):509-518.

4. van Oostrom SH, Nooyens ACJ, van Boxtel MPJ, Verschuren WMM. Long sleep duration is associated with lower cognitive function among middle-age adults - the Doetinchem Cohort Study. Sleep Med. 2018; 41:78-85.

5. Leppävuori A, Pohjasvaara T, Vataja R, Kaste M, Erkinjuntti T. Insomnia in ischemic stroke patients. Cerebrovasc Dis. 2002;14(2):90-97.

6. Mohsenin V, Valor R. Sleep apnea in patients with hemispheric stroke. Arch Phys Med Rehabil. 1995;76(1):71-76.

7. Brown DL. Sleep disorders and stroke. Semin Neurol. 2006;26(1): $117-122$.

8. Lerdal A, Bakken LN, Rasmussen EF, et al. Physical impairment, depressive symptoms and pre-stroke fatigue are related to fatigue in the acute phase after stroke. Disabil Rehabil. 2011;33(4):334-342.
9. Kim J, Kim Y, Yang KI, Kim DE, Kim SA. The Relationship Between Sleep Disturbance and Functional Status in Mild Stroke Patients. Ann Rehabil Med. 2015;39(4):545-552.

10. Glozier N, Moullaali TJ, Sivertsen B, et al. The Course and Impact of Poststroke Insomnia in Stroke Survivors Aged 18 to 65 Years: Results from the Psychosocial Outcomes In StrokE (POISE) Study. Cerebrovasc Dis Extra. 2017;7(1):9-20.

11. Lim J, Dinges DF. A meta-analysis of the impact of short-term sleep deprivation on cognitive variables. Psychol Bull. 2010;136(3):375-389.

12. Goel N, Rao H, Durmer JS, Dinges DF. Neurocognitive consequences of sleep deprivation. Semin Neurol. 2009;29(4):320-339.

13. Eumann Mesas A, López-García E, Rodríguez-Artalejo F. [Sleep duration and functional limitations in older adult]. Med Clin. 2011; 136(12):527-530. Spanish.

14. Friedman EM. Self-Reported Sleep Problems Prospectively Increase Risk of Disability: Findings from the Survey of Midlife Development in the United States. J Am Geriatr Soc. 2016;64(11):2235-2241.

15. Botman S, Moore T, Moiarity Cl PV. Design and estimation for the National Health Interview Survey, 1995-2004. Vital Health Stat 2. 2000;130:1-31.

16. Parsons VL, Moriarity C, Jonas K, Moore TF, Davis KE, Tompkins L. Design and estimation for the national health interview survey, 2006-2015. Vital Health Stat 2. 2014;165:1-53.

17. Appelros P, Stegmayr B, Terént A. A review on sex differences in stroke treatment and outcome. Acta Neurol Scand. 2010;121(6):359-369.

18. Wu MH, Lee S, Su HY, Pai HC. The effect of cognitive appraisal in middle-aged women stroke survivors and the psychological health of their caregivers: a follow-up study. J Clin Nurs. 2015;24(21-22): $3155-3164$.

19. Mesas AE, López-García E, León-Muñoz LM, Guallar-Castillón P, Rodríguez-Artalejo F. Sleep duration and mortality according to health status in older adults. J Am Geriatr Soc. 2010;58(10):1870-1877.

20. Seixas AA, Nunes JV, Airhihenbuwa CO, et al. Linking emotional distress to unhealthy sleep duration: analysis of the 2009 National Health Interview Survey. Neuropsychiatr Dis Treat. 2015;11:2425-2430.

21. Benito-León J, Bermejo-Pareja F, Vega S, Louis ED. Total daily sleep duration and the risk of dementia: a prospective population-based study. Eur J Neurol. 2009;16(9):990-997.

22. $\mathrm{Xu} \mathrm{L}$, Jiang CQ, Lam TH, et al. Short or long sleep duration is associated with memory impairment in older Chinese: the Guangzhou Biobank Cohort Study. Sleep. 2011;34(5):575-580.

23. Duss SB, Seiler A, Schmidt MH, et al. The role of sleep in recovery following ischemic stroke: a review of human and animal data. Neurobiology of Sleep and Circadian Rhythms. 2017;2:94-105.

24. Chung D, Seixas A, Richards SL, et al. The impact of short sleep duration on instrumental activities of daily living (IADL) among stroke survivors [poster abstract]. Available from: https://academic.oup. com/DocumentLibrary/SLEEP/2017\%20Supplement.pdf. Accessed November 26, 2018.
Neuropsychiatric Disease and Treatment

\section{Publish your work in this journal}

Neuropsychiatric Disease and Treatment is an international, peerreviewed journal of clinical therapeutics and pharmacology focusing on concise rapid reporting of clinical or pre-clinical studies on a range of neuropsychiatric and neurological disorders. This journal is indexed on PubMed Central, the 'PsycINFO' database and CAS,

\section{Dovepress}

and is the official journal of The International Neuropsychiatric Association (INA). The manuscript management system is completely online and includes a very quick and fair peer-review system, which is all easy to use. Visit http://www.dovepress.com/testimonials.php to read real quotes from published authors. 